\title{
Origin of subdiffusion of water molecules on cell membrane surfaces
}

SUBJECT AREAS:

MOLECULAR BIOPHYSICS

STATISTICAL PHYSICS, THERMODYNAMICS AND NONLINEAR DYNAMICS

BIOLOGICAL PHYSICS

Received

10 February 2014

Accepted

31 March 2014

Published

17 April 2014

Correspondence and requests for materials should be addressed to K.Y. (yasuoka@mech. keio.ac.jp)

\author{
Eiji Yamamoto', Takuma Akimoto', Masato Yasui ${ }^{2}$ \& Kenji Yasuoka'
}

'Department of Mechanical Engineering, Keio University, 3-4-1 Hiyoshi, Kohoku-ku, Yokohama 223-8522, Japan, ${ }^{2}$ Department of
Pharmacology, School of Medicine, Keio University, 35 Shinanomachi, Shinju-ku, Tokyo 160-8582, Japan.

Water molecules play an important role in providing unique environments for biological reactions on cell membranes. It is widely believed that water molecules form bridges that connect lipid molecules and stabilize cell membranes. Using all-atom molecular dynamics simulations, we show that translational and rotational diffusion of water molecules on lipid membrane surfaces exhibit subdiffusion and aging. Moreover, we provide evidence that both divergent mean trapping time (continuous-time random walk) and long-correlated noise (fractional Brownian motion) contribute to this subdiffusion. These results suggest that subdiffusion on cell membranes causes the water retardation, an enhancement of cell membrane stability, and a higher reaction efficiency.

W ater molecules around cell membranes are important for stability and dynamics of self-assembled lipid structures. Such water molecules form a bridge network that connects lipid molecules ${ }^{1}$. Water molecules form local hydration structures depending on the lipid head groups $\mathrm{s}^{2-4}$ and are weakly aligned by charges on the lipid head group ${ }^{5-8}$. Thus, it is difficult for water molecules to diffuse freely on the surface of the membranes. Qualitatively, translational and rotational motions of water molecules near membranes are slower than those in the bulk ${ }^{9-12}$. Although static properties of such water molecules have been known from experiments, little is known about how water molecules actually diffuse on the membrane surface.

In usual case, diffusion can be characterized by the ensemble-averaged mean square displacement (MSD), i.e., $\left\langle r^{2}(t)\right\rangle=2 D t$, where $D$ is the diffusion constant. However, extensive experimental studies show subdiffusion,

$$
\left\langle\boldsymbol{r}^{2}(t)\right\rangle \simeq K_{\alpha} t^{\alpha} \quad \text { with } \quad 0<\alpha<1,
$$

where $\alpha$ is the subdiffusive exponent and $K_{\alpha}$ is the generalized diffusion constant. There are three well-known stochastic models of subdiffusions with different mechanisms: fractional Brownian motion (FBM) ${ }^{13,14}$, diffusion on a fractal lattice ${ }^{15}$, and continuous-time random walk $(\mathrm{CTRW})^{16}$. Because these models have different physical nature, revealing the origin is significant to understand physical properties ${ }^{17,18}$. In particular, the physical origin of subdiffusion in living cells has been extensively studied ${ }^{19-23}$. Previously, water molecules near the surface of lipid membranes were found to show transient subdiffusion ${ }^{24,25}$, and water molecules exhibit subdiffusion on the surfaces ${ }^{12}$. However, the origin of this water subdiffusion remains unclear.

In general, it is difficult to identify the mechanism underlying subdiffusion. Ergodic and aging properties play an important role in clarifying the physical origin. It is known that FBM motion is ergodic, whereas under confinement a power-law relaxation of time-averaged mean square displacement occurs for a model related to $\mathrm{FBM}^{26,27}$. Since ordinary ergodicity, where the time averages are equal to the ensemble average, holds for FBM, and diffusion on a fractal lattice ${ }^{28,29}$, the dominant feature of CTRW with a divergent mean trapping time is aging and weak ergodicity breaking ${ }^{30-33}$. Such phenomena are also observed in a range of stochastic models different from the CTRW such as random walk with static disorder ${ }^{34}$, random walks with correlated waiting times ${ }^{35,36}$, spatially correlated random walks ${ }^{37}$, aging walks ${ }^{38}$, and stored-energy-driven Lévy flight ${ }^{39}$.

Divergence of the mean trapping time is attributed to a power law in the trapping-time distribution. Power laws are often observed in biological phenomena ${ }^{21,40-42}$. One of the mechanisms generating a power-law trapping-time distribution is a random-energy landscape ${ }^{30}$. There are many binding sites in one-dimensional diffusion along DNA, two-dimensional diffusion on the plasma membrane, and three-dimensional diffusion in the cytoplasm ${ }^{43}$. If the potential depth of each binding site is randomly distributed according to the exponential distribution, the distribution of the trapping times for which particles are trapped in the binding sites follows a power law ${ }^{44}$.

Here, we perform molecular dynamics (MD) simulations on two systems of water molecules plus membranes, of either palmitoyl-oleoyl-phosphocholine (POPC) or palmitoyl-oleoyl-phosphatidylethanolamine (POPE), at 

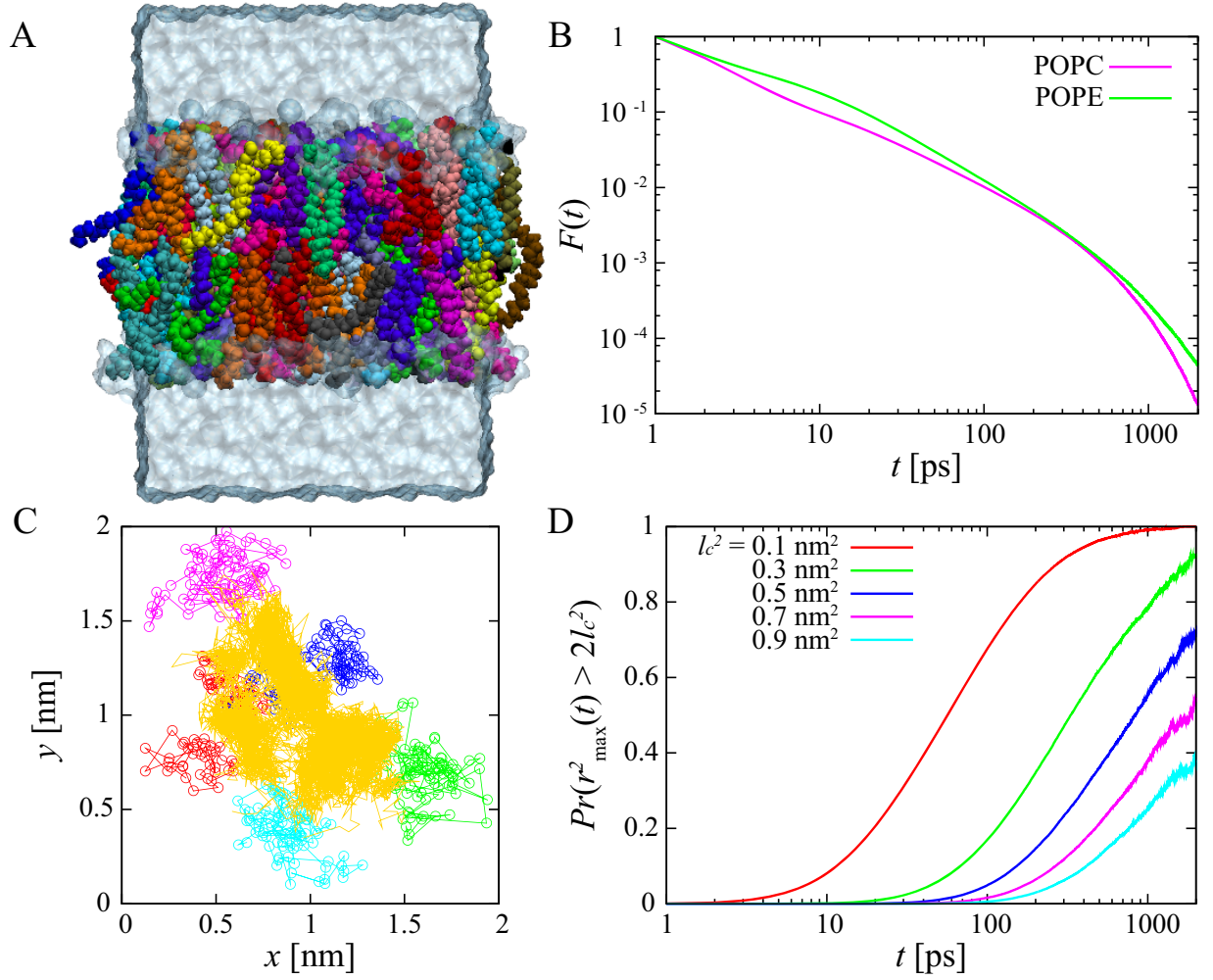

Figure $1 \mid$ Diffusion of water molecules on lipid membrane surfaces. (A) Configuration of POPC bilayer. Each color represents a different phospholipid. Explicit water molecules correspond to the upper and lower transparent coatings. (B) Survival provability of residence time of water molecules on the membrane surfaces. (C) Lateral trajectory of a water molecule (yellow) tracked for 9 ns on the POPE membrane surface. Circles with lines represent trajectories of the $\mathrm{C} 2$ atom (see Fig. S1) in different lipid molecules. (D) Fraction of water molecules traversing a certain distance $l_{c}$ as a function of the residence time $t$. Each color represents different $l_{c}$ values (see key legend).

the temperature $310 \mathrm{~K}$ to investigate the diffusion of water molecules on the membrane surface (Fig. 1A). Here, we report on subdiffusion of water molecules on the membrane surfaces. Furthermore, we show that the subdiffusion is attributed to the divergent mean trapping time and anti-correlated noise, i.e., a combination of CTRW and FBM scenarios. We confirm there are no qualitative differences about subdiffusive behavior despite of the different water structure at ammonium head groups of POPC and $\mathrm{POPE}^{2}$.

\section{Results}

Diffusions of water molecules on membrane surfaces. Water molecules forming the bridges connecting lipid molecules on the membrane surface do not diffuse. This bridge is formed by hydrogen bonds between the water molecules and head groups of the lipid molecules. These hydrogen bond interactions create a complicated and random potential surface over the membrane. To investigate the diffusivity of water molecules on the membrane surfaces, we define surface water molecules as water molecules for which the oxygens remain continuously within interatomic distances of $0.35 \mathrm{~nm}$ from atoms (oxygen, phosphorus, nitrogen, and carbon atoms) in the lipid molecules. In what follows, we use trajectories of the water and lipid molecules where the position of the center of mass of the membrane is subtracted.

First, we consider the survival probability of residence time of water molecules on the membrane surface, where the residence time is defined as the duration for which a water molecule remains on the membrane surface. The survival provability is defined by $F(t)=\int_{t}^{\infty} P\left(t^{\prime}\right) d t^{\prime}$, where $P(t)$ is a probability density function (PDF). The PDFs of the residence times follow power-law distributions with exponential cutoffs in their tails (see Fig. S2). Mean residence times on POPC and POPE bilayers are $7.0 \pm 36$ ps and $9.3 \pm$
$42 \mathrm{ps}$, respectively. Although almost all of the water molecules pass on the membrane surfaces without binding to the lipid molecules, we are interested in the water molecules diffusing on lipid membrane surfaces. This is because water molecules residing on the surface for long time can contribute to membrane stability by making water bridges and may have important role in many physiological processes.

Figure 1C shows a lateral trajectory of a water molecule residing on the POPE bilayer surface for 9 ns. Surprisingly, water molecules do diffuse widely on the membrane surface while residing on it. In other words, water bridges connecting lipid molecules are not static but dynamical. Indeed, diffusion distances on the membrane surface lengthen with increasing residence times. Figure 1D shows the probability that the maximal excursion distance for water molecules is greater than $l_{c}, \operatorname{Pr}\left(r_{\max }^{2}(t)>2 l_{c}^{2}\right)$, where the maximal excursion distance is defined by $r_{\max }(t)=\max \left\{r\left(t^{\prime}\right): 0 \leq t^{\prime} \leq t\right\}$ with $r(t)=\sqrt{x(t)^{2}+y(t)^{2}}$. About $40 \%$ of water molecules that reside on the membrane surface over 600 ps can diffuse above $0.5 \mathrm{~nm}^{2}$. This implies that water molecules can diffuse beyond a lipid molecule in the membranes, because the area per lipid is about $0.5-0.7 \mathrm{~nm}^{2}$. Some water molecules diffuse by interchanging the water bridge while remaining on the membrane surface. Thus, we found a water-bridge interchange dynamics for the first time.

Translational and rotational subdiffusion of water molecules. To investigate the diffusion of water molecules on the membrane surfaces, we consider translational as well as rotational diffusions of the water molecules. The ensemble-averaged lateral translational MSD (tMSD) is defined as

$$
\left\langle l^{2}(t)\right\rangle=\frac{1}{2}\left\langle\left\{x\left(t+t_{0}\right)-x\left(t_{0}\right)\right\}^{2}+\left\{y\left(t+t_{0}\right)-y\left(t_{0}\right)\right\}^{2}\right\rangle,
$$


A

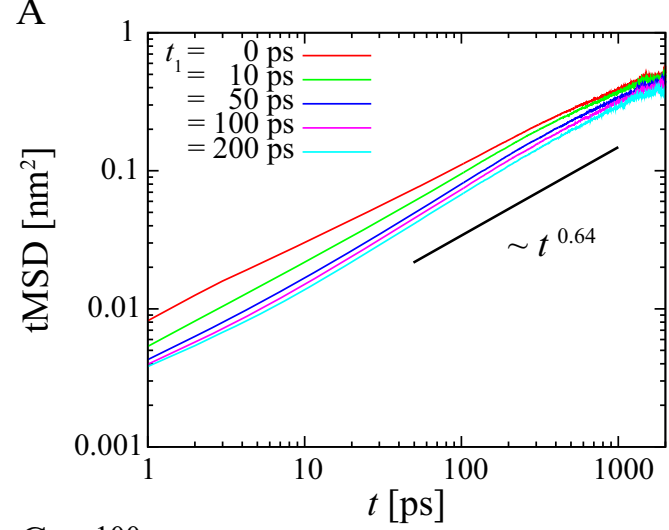

$\mathrm{C}$

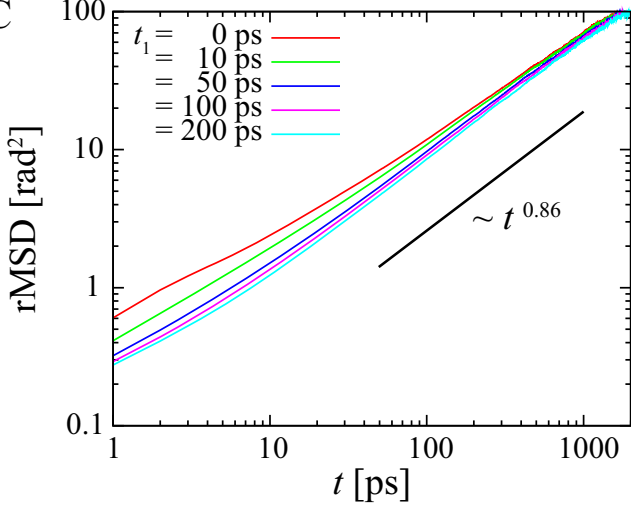

B

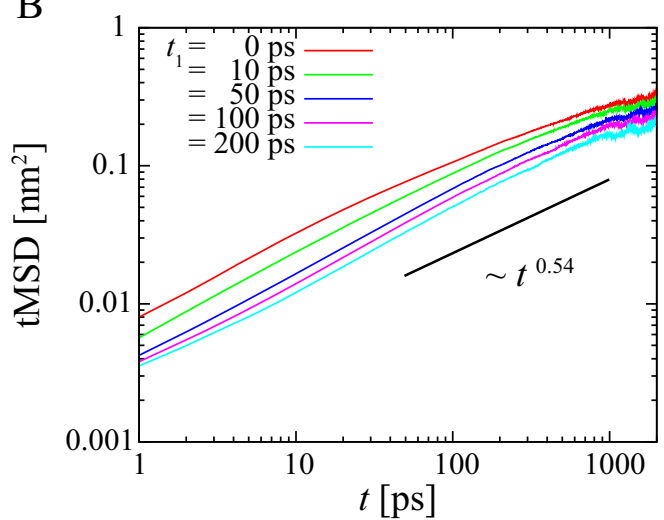

$\mathrm{D}$

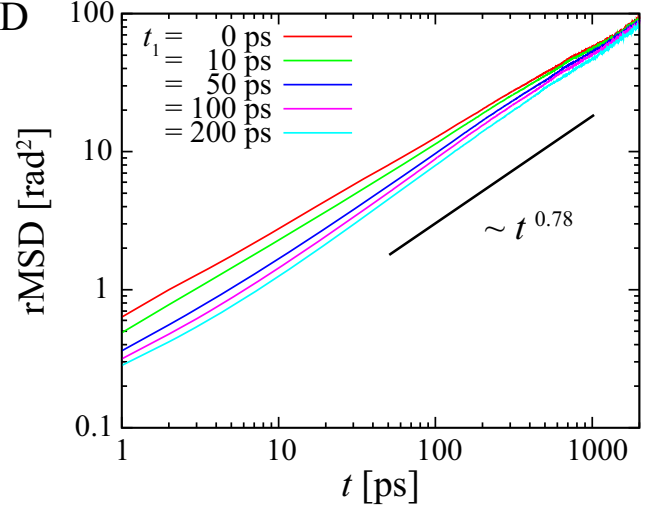

Figure 2 Translational and rotational subdiffusion of water molecules. (A) Ensemble-averaged tMSD and (C) rMSD of water molecules on the POPC membrane surface. (B) and (D) are the tMSD and rMSD on the POPE membrane surface. The slope of the solid lines are fitted in the time interval from 50 to $1000 \mathrm{ps}$ for $t_{1}=200 \mathrm{ps}$. The different colored lines correspond to different measurement starting times $t_{1}$.

where $t_{0}$ is the time when water molecules enter the membrane surfaces and $\langle\ldots\rangle$ is the average with respect to captured and reflected the water molecules impinging on the membrane surfaces. If exiting from the membrane surfaces, water molecules are excluded from the ensemble. In considering rotational diffusion, we define $\delta \theta(t)=\cos ^{-1}(\vec{\mu}(t) \cdot \vec{\mu}(t+\delta t))$ and direction $\vec{p}(t) \equiv \vec{\mu}(t) \times \vec{\mu}(t+\delta t)$, where $\vec{\mu}(t)$ is the dipole vector of a water molecule at time $t$. The vector $\vec{\varphi}(t) \equiv \int_{t_{0}}^{t_{0}+t} \delta \theta\left(t^{\prime}\right) \vec{p}\left(t^{\prime}\right) d t^{\prime}$ gives us the trajectory representing the rotational motion. Then, the ensemble-averaged rotational mean-squared displacement $(\mathrm{rMSD})^{45}$ is given by

$$
\left\langle\varphi^{2}(t)\right\rangle=\left\langle|\vec{\varphi}(t)-\vec{\varphi}(0)|^{2}\right\rangle .
$$

In CTRW, the MSD is suppressed with increase of the starting time $t_{1}$ of a measurement ${ }^{46}$. This behavior is called aging. To investigate aging, we consider the dependence of the MSDs on the starting time of a measurement. Here, we consider $\operatorname{tMSD}\left(t ; t_{1}\right)=\left\langle\left\{x\left(t+t_{0}\right.\right.\right.$ $\left.\left.\left.+t_{1}\right)-x\left(t_{0}+t_{1}\right)\right\}^{2}+\left\{y\left(t+t_{0}+t_{1}\right)-y\left(t_{0}+t_{1}\right)\right\}^{2}\right\rangle / 2$ and $\operatorname{rMSD}\left(t ; t_{1}\right)=\left\langle\left|\vec{\varphi}\left(t+t_{0}+t_{1}\right)-\vec{\varphi}\left(t_{0}+t_{1}\right)\right|^{2}\right\rangle$, where $t_{1}$ corresponds to times after entering the membrane surface at $t_{0}$. Figure 2 shows the MSDs measured after time $t_{1}$ from 0 to 200 ps. Translational motions of water molecules exhibit subdiffusion as in diffusion of lipid molecules ${ }^{42,47-50}$. Whereas the subdiffusive exponents in the tMSDs decrease as time $t$ increases, the rMSDs show subdiffusion with a constant subdiffusive exponent. For tMSD and rMSD, water molecules on POPC bilayers are faster than those on POPE bilayers. This is because hydrogen bonds between choline groups and water molecules in POPC bilayers are weaker than those in POPE bilayers because methyl groups are present in the choline group of POPC. Moreover, as seen in Fig. 2, both tMSD and rMSD depend on the starting time of a measurement $t_{1}$. Both MSDs become smaller the later $t_{1}$ becomes. For $t_{1}>50 \mathrm{ps}$, unlike CTRW, MSDs do not strongly depend on $t_{1}$. Therefore, this aging will be affected by a non-equilibrium initial condition when water molecules attach to the membrane surfaces. We note that MSDs show aging even when non-equilibrium initial conditions are skipped (see Fig. S3).

Origin of subdiffusive motion of water molecules. To clarify the origin of subdiffusive motions of water molecules on membrane surfaces, we perform a mean maximal excursion (MME) analysis ${ }^{18}$. The MME analysis provides us an information on the physical nature of the underlying subdiffusive processes by using trajectories only. In Fig. 3A, the translational and rotational MSDs, $\left\langle l^{2}(t)\right\rangle$ and $\left\langle\varphi^{2}(t)\right\rangle$, and the MME second moments, $\left\langle l^{2}(t)_{\max }\right\rangle$ and $\left\langle\varphi^{2}(t)_{\max }\right\rangle$, grow sublinearly with time, where $\left\langle l^{2}(t)_{\max }\right\rangle$ and $\left\langle\varphi^{2}(t)_{\max }\right\rangle$ are the ensemble averages of $l_{\max }(t)=\max \left\{l\left(t^{\prime}\right): 0 \leq t^{\prime} \leq t\right\}$ and $\varphi_{\max }(t)$ $=\max \left\{\varphi\left(t^{\prime}\right): 0 \leq t^{\prime} \leq t\right\}$, respectively. For about $t>30 \mathrm{ps}$, the subdiffusive exponents of MSDs are almost the same as those of the MME second moment. This result suggests that a fractal or CTRW feature appears over relatively large-time intervals. Moreover, Fig. 3B shows that the regular moment ratios $\left\langle l^{4}(t)\right\rangle /$ $\left\langle l^{2}(t)\right\rangle^{2}$ and $\left\langle\varphi^{4}(t)\right\rangle /\left\langle\varphi^{2}(t)\right\rangle^{2}$ fluctuate above 2 except for $\left\langle\varphi^{4}(t)\right\rangle /$ $\left\langle\varphi^{2}(t)\right\rangle^{2}$ around $1000 \mathrm{ps}$ and that the MME moment ratios $\left\langle l^{4}(t)_{\max }\right\rangle\left\langle\left\langle l^{2}(t)_{\max }\right\rangle^{2}\right.$ and $\left\langle\varphi^{4}(t)_{\max }\right\rangle /\left\langle\varphi^{2}(t)_{\max }\right\rangle^{2}$ fluctuate above 1.49. This result suggests CTRW scenario and excludes FBM and fractal scenarios. Figure $3 \mathrm{C}$ shows that the probability for water molecules to be in a sphere of growing radius $r_{0} t^{\alpha / 2}$ is almost constant over $t$, while for rotational diffusions, the probability below 20 ps increases because of a change in the subdiffusive exponent. This result suggests CTRW or FBM scenarios and excludes fractal scenario. The above results are summarized in Table 1 . These results strongly support the CTRW scenario for large-time intervals. 
A

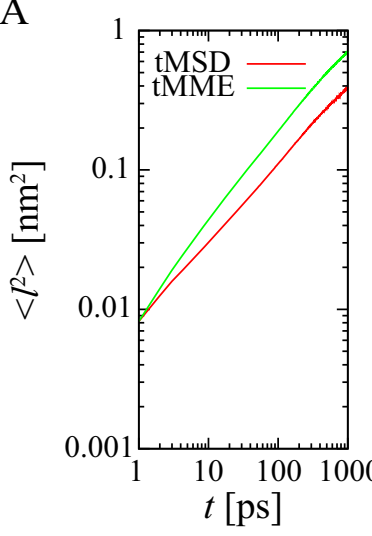

B

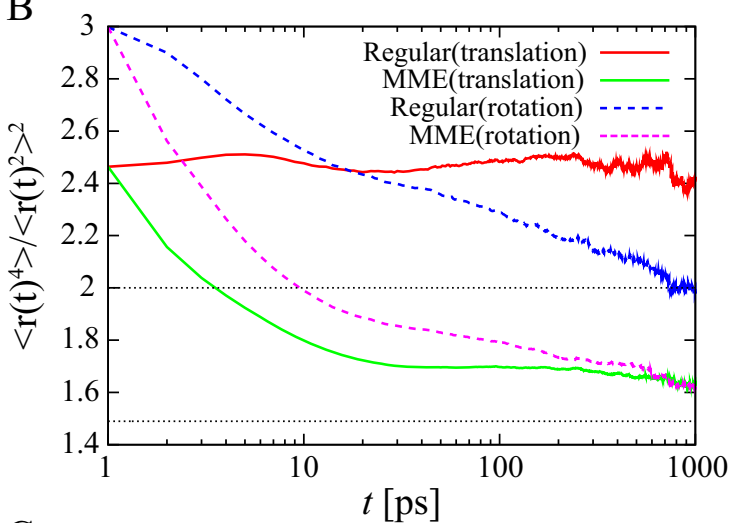

$\mathrm{C}$

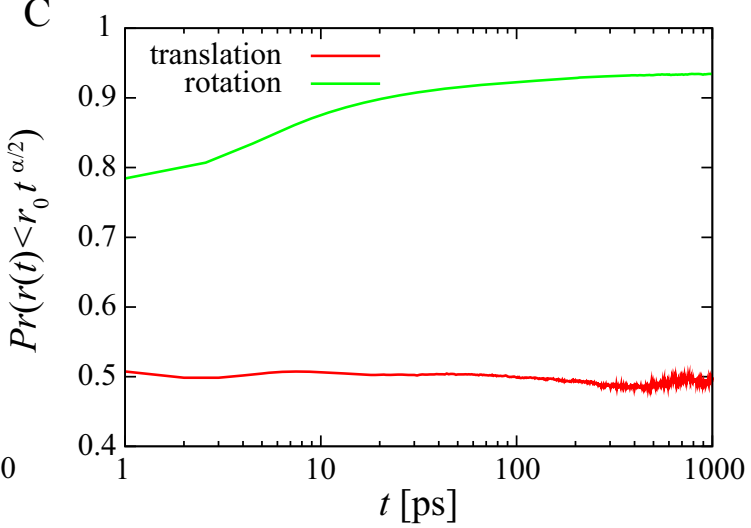

Figure 3 Quantitative analysis of trajectories of water molecules on the POPC membrane surface. (A) MSD and second MME moment as functions of time $t$ for translational and rotational diffusions. (B) Regular and MME moment ratios for translational and rotational motions. Horizontal lines are ratios 2 and 1.49. (C) Probability of water molecules to be in a sphere of growing radius $r_{0} t^{\alpha / 2}$. The value of $\alpha$ is based on fitted values 0.56 and 0.76 for translational and rotational motions in the time interval from 10 to $1000 \mathrm{ps}$ for $t_{1}=0 \mathrm{ps}$, respectively.

To validate the CTRW scenario, we consider the time-averaged mean square displacements (TAMSDs) defined by $\overline{\delta^{2}(\Delta ; t)}=\left(\overline{\delta_{x}^{2}(\Delta ; t)}+\right.$ $\left.\overline{\delta_{y}^{2}(\Delta ; t)}\right) / 2$ and $\overline{\delta_{\varphi}^{2}(\Delta ; t)}=\frac{1}{t-\Delta} \int_{0}^{t-\Delta}\left|\vec{\varphi}\left(t^{\prime}+\Delta\right)-\vec{\varphi}\left(t^{\prime}\right)\right|^{2} d t^{\prime}$ for translational and rotational motions, respectively, where $t$ is the measurement time and $\overline{\delta_{x}^{2}(\Delta ; t)}=\int_{0}^{t-\Delta}\left\{x\left(t^{\prime}+\Delta\right)-x\left(t^{\prime}\right)\right\}^{2} d t^{\prime} /$ $(t-\Delta)$. TAMSDs for trajectories of water molecules residing on the surface of the membrane longer than 2000 ps for both translational and rotational motions are shown in Figs. $4 \mathrm{~A}$ and $4 \mathrm{~B}$, respectively. Unlike CTRW, where the TAMSD grows linearly with $\Delta$, TAMSDs do not show a linear scaling over short-time durations. Because the TAMSD shows subdiffusion in FBM, i.e., sublinear scaling of $\Delta$, translational and rotational motions have a FBM characteristic over short-time durations of $\Delta$. However, rotational TAMSDs

Table 1 | Summary of confirming the origin of subdiffusive motions

\begin{tabular}{ll} 
Analysis & Translational \\
\hline Second moment (regular, MME) & Fractals or CTRW $\left[\propto t^{\alpha}, \propto t^{\alpha}\right]$ \\
Ratio (regular, MME) & CTRW $[>2,>1.49]$ \\
Growing spheres & CTRW or FBM $\left[\operatorname{Pr}\left(r \leq r_{0} t^{\alpha / 2}, t\right)=A_{0}\right]$ \\
TAMSD & FBM
\end{tabular}

Analysis

Rotational

Second moment (regular, MME)

Ratio (regular, MME)

Growing spheres

TAMSD show normal diffusion (linear scaling of $\Delta$ ) as expected by CTRW, whereas translational TAMSDs do not show normal diffusion. The mean rotational TAMSDs crossover from sublinear to linear (see Fig. S5). The crossover points at around $10 \mathrm{ps}$ are coincident with the relaxation time for the orientational correlation functions of water molecules on the membrane surfaces ${ }^{12}$. Because the sublinear growth of the TAMSDs suggests FBM, the dynamics of water molecules will be affected by viscoelasticity.

Figures $4 \mathrm{C}$ and $4 \mathrm{D}$ show the aging plots for translational and rotational TAMSDs on the POPC membrane surface, i.e., the ensemble average of the TAMSD as a function of the measurement time $t$, for different measurement starting times $t_{1}$. Whereas the ensemble averages of translational and rotational TAMSDs show power-law decays: $\overline{\left\langle\delta^{2}(\Delta ; t)\right\rangle} \propto t^{-\gamma_{1}}$ and $\overline{\left\langle\delta_{\varphi}^{2}(\Delta ; t)\right\rangle} \propto t^{-\gamma_{2}}$ for $t_{1}<$ $50 \mathrm{ps}$, those do not decay for $t_{1}>50 \mathrm{ps}$. In CTRW, the ensemble

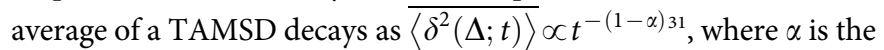
power-law exponent for the trapping-time PDF. However, recently, it is shown that CTRW with strong noisy fluctuations do not show the aging of TAMSD, whereas MSD still shows aging ${ }^{51}$. Thus, the power-law decays of ensemble average of TAMSDs for $t_{1}<50$ ps are attributed to non-equilibrium initial conditions of water molecules on the membrane surfaces. This is because mean velocity of bulk water molecules is higher than those on the membrane surfaces. We note that MSDs show aging in our simulations even when an initial non-equilibrium state is skipped (see Fig. S3).

Together with the MME analysis, it is physically reasonable to consider that the origin of the observed subdiffusion is a combination of CTRW and FBM. Although we do not provide a direct evidence of aging effect, results in noisy CTRW ${ }^{51}$ assist a suggestion that aging due to CTRW is inherent in water dynamics on the membrane 

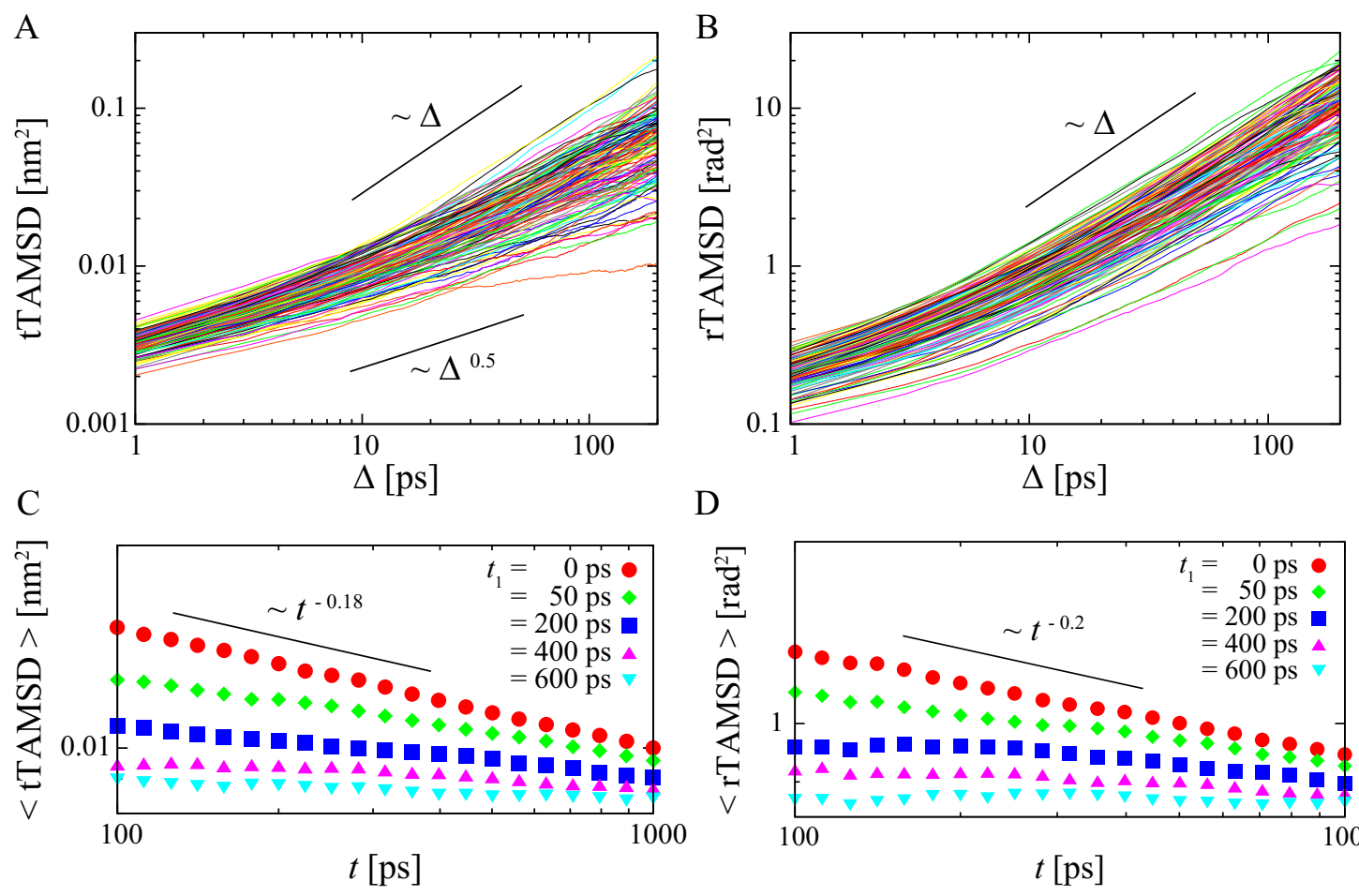

$\mathrm{D}$

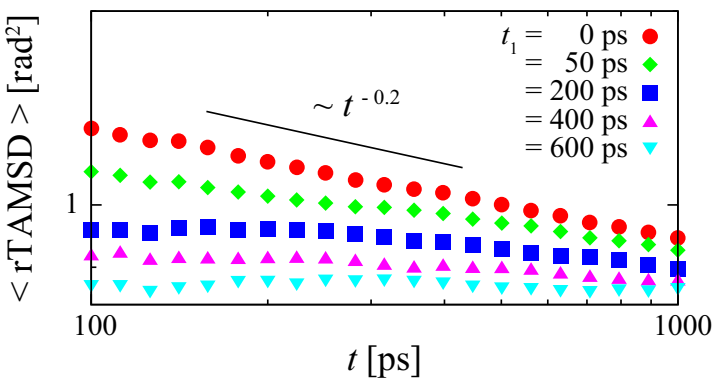

Figure $4 \mid$ Time-averaged mean square displacements analysis. (A) Translational and (B) rotational TAMSDs of water molecules on the POPC membrane surface. The different colored lines show 128 trajectories of water molecules. (C) Aging plot for translational and (D) rotational TAMSD for $\Delta$ $=10$ ps. The different colored symbols correspond to different measurement starting times $t_{1}$. For reference, the power-law decays are represented by solid lines.

surfaces. We note that non-equilibrium conditions of water molecules on the membrane surface are compatible with an equilibration of the total system. As shown in the supporting information (Fig. S11, S12), total systems are equilibrated whereas TAMSDs show apparent aging (see aging plot in Fig. 4). This apparent inconsistency can be resolved by dissociation of water molecules from the membrane surfaces. In fact, because water molecules can dissociate from the membrane surfaces and the mean residence time is finite, the system can be equilibrated.

The distribution of waiting times contributes to CTRW arising from random binding and unbinding of water molecules from the lipid surface. Moreover, translational motions of water molecules forming the water bridge are affected by lipid motions in lipid membranes which are governed by FBM motions ${ }^{42,49,50}$. Unlike CTRW, where a trapped state simply does not move, it is difficult to estimate exact trapping times in such situations. Thus, we do not observe power-law trapping-time distributions in lateral motions (see Fig. S8) because water molecules on the membrane surface can move during a trapped state. We also confirmed that there are no significant differences in the water behavior on both POPC and POPE lipid membranes (see Fig. S3-S9). Subdiffusion and aging are also observed with using other force filed, thermostat, and barostat (see Fig. S10, the detail of MD simulations is written in our previous paper $\left.^{12}\right)$.

\section{Discussion}

In summary, we have shown that water molecules on membrane surfaces can diffuse laterally while connected as part of a bridging network to lipid molecules in membrane. This interchanging dynamics in the water bridge network can be described by CTRW. Furthermore, we have found translational and rotational subdiffusion of water molecules on the membrane surfaces. These subdiffusions originate from a combination of CTRW and FBM, which are attributed to long-time trapping by the membrane surface and viscoelasticity of lipid bilayers, respectively. Such a subdiffusive process has been observed in experiments of intracellular transport of insulin granules ${ }^{23}$.

What is a biological significance of subdiffusion and aging phenomena of water molecules on cell membrane surfaces? Because subdiffusion and aging mean the diffusivity decreases as time passes, these phenomena cause water retardation and increase the chance of water binding on the membrane surfaces. As a result, water molecules form bridges that connect lipid molecules and stabilize cell membranes. Moreover, the water retardation may contribute to higher efficiency of biological reactions on cell membranes. To recognize and bind to a target, biomolecules diffuse slowly around the target, and may be guided by the behavior of water molecules in the target vicinity ${ }^{52}$. For example, water retardation around a metalloenzyme active site assists enzyme-substrate interactions ${ }^{53}$. In a stochastic model, the probability of finding a nearby target is explicitly increased by subdiffusion ${ }^{54}$. Biological reactions such as ligand-receptor interactions and enzymatic reactions occur on cell membranes. Those depend upon encounters between biomolecules, and the structure and dynamics of water molecules in the hydration layer on the membranes. The water environment around the cell membranes produced by the anomalous diffusion of water molecules may have biological significance.

\section{Methods}

MD simulations. To clarify water molecule dynamics on the lipid membrane surface, MD simulations of pure POPC or POPE bilayers were performed. Each lipid bilayer system consisted of 128 lipids (64 for each leaflet) and 7,680 TIP3P water molecules (see Fig. 1(A)). The CHARMM36 $6^{55}$ force field was used for the lipids. The TIP3P water model modified for the CHARMM force field ${ }^{56}$ was used because the CHARMM36 force field was developed based on the TIP3P water models. Although the diffusion constant of TIP3P water model is higher than the experimental values, it reproduces the first-shell hydration and the energetics of liquid water ${ }^{57}$. The bond lengths involving the hydrogen atoms were constrained to equilibrium lengths using the SHAKE method. The direct sum and Lennard-Jones interactions were smoothly truncated at a cutoff distance of $1.2 \mathrm{~nm}$, using a switching function that becomes effective at $1.0 \mathrm{~nm}$. The particle-mesh Ewald method was used for calculations of 
electrostatic interactions. Before MD simulations, energy minimization was performed using a conjugate gradient algorithm to remove the bad contacts of the initial configuration. Simulations were performed under constant NPT (number of particles, pressure, and temperature) with temperature $310 \mathrm{~K}$ and pressure $0.1 \mathrm{MPa}$. For temperature and pressure control, a Langevin thermostat and piston were used with a damping coefficient of $1 \mathrm{ps}^{-1}$ and a collision period of $0.2 \mathrm{ps}$, respectively. The three orthogonal dimensions of the periodic cell were allowed to change independently. Each simulation ran for 240 ns under 2.0 fs time-step increments; coordinates were recorded every $1.0 \mathrm{ps}$. The final system sizes of MD simulations of POPC and POPE bilayers are $6.6 \times 6.6 \times 8.8 \mathrm{~nm}^{3}$ and $6.1 \times 6.1 \times 10.2 \mathrm{~nm}^{3}$ respectively. The properties of each system were analyzed over the trajectories of the final 130 ns of each system. All MD simulations were performed using NAMD2.9 software ${ }^{58}$

1. Pasenkiewicz-Gierula, M., Takaoka, Y., Miyagawa, H., Kitamura, K. \& Kusumi, A Hydrogen bonding of water to phosphatidylcholine in the membrane as studied by a molecular dynamics simulation: location, geometry, and lipid-lipid bridging via hydrogen-bonded water. J. Phys. Chem. A 101, 3677-3691 (1997).

2. Damodaran, K. V. \& Merz, K. M. Jr. Head group-water interactions in lipid bilayers: a comparison between $\mathrm{dmpc}$-and dlpe-based lipid bilayers. Langmuir 9 1179-1183 (1993).

3. Alper, H. E., Bassolino-Klimas, D. \& Stouch, T. R. The limiting behavior of water hydrating a phospholipid monolayer: a computer simulation study. J. Chem. Phys. 99, 5547 (1993).

4. Lopez, C. F., Nielsen, S. O., Klein, M. L. \& Moore, P. B. Hydrogen bonding structure and dynamics of water at the dimyristoylphosphatidylcholine lipid bilayer surface from a molecular dynamics simulation. J. Phys. Chem. B $\mathbf{1 0 8}$ 6603-6610 (2004)

5. Nagata, Y. \& Mukamel, S. Vibrational sum-frequency generation spectroscopy at the water/lipid interface: molecular dynamics simulation study. J. Am. Chem. Soc. 132, 6434-6442 (2010)

6. Mondal, J. A., Nihonyanagi, S., Yamaguchi, S. \& Tahara, T. Structure and orientation of water at charged lipid monolayer/water interfaces probed by heterodyne-detected vibrational sum frequency generation spectroscopy. J. Am. Chem. Soc. 132, 10656-10657 (2010).

7. Chen, X., Hua, W., Huang, Z. \& Allen, H. C. Interfacial water structure associated with phospholipid membranes studied by phase-sensitive vibrational sum frequency generation spectroscopy. J. Am. Chem. Soc. 132, 11336-11342 (2010).

8. Mondal, J. A., Nihonyanagi, S., Yamaguchi, S. \& Tahara, T. Three distinct water structures at a zwitterionic lipid/water interface revealed by heterodyne-detected vibrational sum frequency generation. J. Am. Chem. Soc. 134, 7842-7850 (2012).

9. Róg, T., Murzyn, K. \& Pasenkiewicz-Gierula, M. The dynamics of water at the phospholipid bilayer surface: a molecular dynamics simulation study. Chem. Phys. Lett. 352, 323-327 (2002).

10. Bhide, S. Y. \& Berkowitz, M. L. Structure and dynamics of water at the interface with phospholipid bilayers. J. Chem. Phys. 123, 224702 (2005).

11. Murzyn, K., Zhao, W., Karttunen, M., Kurdziel, M. \& Róg, T. Dynamics of water at membrane surfaces: Effect of headgroup structure. Biointerphases 1, 98 (2006).

12. Yamamoto, E., Akimoto, T., Hirano, Y., Yasui, M. \& Yasuoka, K. Power-law trapping of water molecules on the lipid-membrane surface induces water retardation. Phys. Rev. E 87, 052715 (2013).

13. Kolmogorov, A. N. The wiener spiral and some other interesting curves in hilbert space. Dokl Acad Sci USSR 26, 115 (1940).

14. Mandelbrot, B. B. \& Van Ness, J. W. Fractional brownian motions, fractional noises and applications. SIAM Rev. 10, 422-437 (1968).

15. Ben-Avraham, D. \& Havlin, S. Diffusion and reactions in fractals and disordered systems (Cambridge Univ Pr, 2000).

16. Metzler, R. \& Klafter, J. The random walk's guide to anomalous diffusion: a fractional dynamics approach. Phys. Rep. 339, 1-77 (2000).

17. Mason, T. G. \& Weitz, D. A. Optical measurements of frequency-dependent linear viscoelastic moduli of complex fluids. Phys. Rev. Lett. 74, 1250-1253 (1995).

18. Tejedor, V. et al. Quantitative analysis of single particle trajectories: mean maximal excursion method. Biophys. J. 98, 1364-1372 (2010).

19. Golding, I. \& Cox, E. C. Physical nature of bacterial cytoplasm. Phys. Rev. Lett. 96, 098102 (2006).

20. Jeon, J.-H. et al. In Vivo anomalous diffusion and weak ergodicity breaking of lipid granules. Phys. Rev. Lett. 106, 048103 (2011).

21. Weigel, A. V., Simon, B., Tamkun, M. M. \& Krapf, D. Ergodic and nonergodic processes coexist in the plasma membrane as observed by single-molecule tracking. Proc. Natl. Acad. Sci. USA 108, 6438-6443 (2011).

22. Barkai, E., Garini, Y. \& Metzler, R. Strange kinetics of single molecules in living cells. Phys. Today 65, 29-35 (2012)

23. Tabei, S. M. A. et al. Intracellular transport of insulin granules is a subordinated random walk. Proc. Natl. Acad. Sci. USA 110, 4911-4916 (2013).

24. Das, J., Flenner, E. \& Kosztin, I. Anomalous diffusion of water molecules in hydrated lipid bilayers. J. Chem. Phys. 139, 065102 (2013).

25. von Hansen, Y., Gekle, S. \& Netz, R. R. Anomalous anisotropic diffusion dynamics of hydration water at lipid membranes. Phys. Rev. Lett. 111, 118103 (2013).

26. Jeon, J.-H. \& Metzler, R. Inequivalence of time and ensemble averages in ergodic systems: Exponential versus power-law relaxation in confinement. Phys. Rev. E 85, 021147 (2012).
27. Jeon, J.-H., Leijnse, N., Oddershede, L. B. \& Metzler, R. Anomalous diffusion and power-law relaxation of the time averaged mean squared displacement in wormlike micellar solutions. New J. Phys. 15, 045011 (2013).

28. Deng, W. \& Barkai, E. Ergodic properties of fractional brownian-langevin motion Phys. Rev. E 79, 011112 (2009).

29. Jeon, J. H. \& Metzler, R. Analysis of short subdiffusive time series: scatter of the time-averaged mean-squared displacement. J. Phys. A: Math. Theor. 43, 252001 (2010).

30. Bouchaud, J. P. Weisshashimotonilsson2003. J. Phys. I 2, 1705-1713 (1992).

31. He, Y., Burov, S., Metzler, R. \& Barkai, E. Random time-scale invariant diffusion and transport coefficients. Phys. Rev. Lett. 101, 058101 (2008).

32. Lubelski, A., Sokolov, I. M. \& Klafter, J. Nonergodicity mimics inhomogeneity in single particle tracking. Phys. Rev. Lett. 100, 250602 (2008).

33. Miyaguchi, T. \& Akimoto, T. Ergodic properties of continuous-time random walks: Finitesize effects and ensemble dependences. Phys. Rev. E 87, 032130 (2013).

34. Miyaguchi, T. \& Akimoto, T. Intrinsic randomness of transport coefficient in subdiffusion with static disorder. Phys. Rev. E 83, 031926 (2011).

35. Tejedor, V. \& Metzler, R. Anomalous diffusion in correlated continuous time random walks. J. Phys. A 43, 082002 (2010).

36. Magdziarz, M., Metzler, R., Szczotka, W. \& Zebrowski, P. Correlated continuoustime random walks in external force fields. Phys. Rev. E 85, 051103 (2012).

37. Cherstvy, A. G., Chechkin, A. V. \& Metzler, R. Anomalous diffusion and ergodicity breaking in heterogeneous diffusion processes. New J. Phys. 15, 083039 (2013).

38. Lomholt, M. A., Lizana, L., Metzler, R. \& Ambjörnsson, T. Microscopic origin of the logarithmic time evolution of aging processes in complex systems. Phys. Rev. Lett. 110, 208301 (2013).

39. Akimoto, T. \& Miyaguchi, T. Distributional ergodicity in stored-energy-driven lévy flights. Phys. Rev. E 87, 062134 (2013).

40. Wong, I. Y. et al. Anomalous diffusion probes microstructure dynamics of entangled F-Actin networks. Phys. Rev. Lett. 92, 178101 (2004).

41. van Hijkoop, V. J., Dammers, A. J., Malek, K. \& Coppens, M.-O. Water diffusion through a membrane protein channel: A first passage time approach. J. Chem. Phys. 127, 085101 (2007).

42. Akimoto, T., Yamamoto, E., Yasuoka, K., Hirano, Y. \& Yasui, M. Non-gaussian fluctuations resulting from power-law trapping in a lipid bilayer. Phys. Rev. Lett. 107, 178103 (2011)

43. Saxton, M. J. A biological interpretation of transient anomalous subdiffusion. i. qualitative model. Biophys. J. 92, 1178-1191 (2007).

44. Bardou, F., Bouchaud, J. P., Aspect, A. \& Tannoudji, C. C. Lévy statistics and laser cooling: how rare events bring atoms to rest (Cambridge University Press, 2002).

45. Mazza, M. G., Giovambattista, N., Starr, F. W. \& Stanley, H. E. Relation between rotational and translational dynamic heterogeneities in water. Phys. Rev. Lett. 96, 057803 (2006).

46. Barkai, E. Aging in subdiffusion generated by a deterministic dynamical system. Phys. Rev. Lett. 90, 104101 (2003).

47. Weiss, M., Hashimoto, H. \& Nilsson, T. Anomalous protein diffusion in living cells as seen by fluorescence correlation spectroscopy. Biophys. J. 84, 4043 (2003)

48. Flenner, E., Das, J., Rheinstädter, M. C. \& Kosztin, I. Subdiffusion and lateral diffusion coefficient of lipid atoms and molecules in phospholipid bilayers. Phys. Rev. E 79, 011907 (2009).

49. Kneller, G. R., Baczynski, K. \& Pasenkiewicz-Gierula, M. Communication: Consistent picture of lateral subdiffusion in lipid bilayers: Molecular dynamics simulation and exact results. J. Chem. Phys. 135, 141105 (2011).

50. Jeon, J.-H., Monne, H. M.-S., Javanainen, M. \& Metzler, R. Anomalous diffusion of phospholipids and cholesterols in a lipid bilayer and its origins. Phys. Rev. Lett. 109, 188103 (2012).

51. Jeon, J.-H., Barkai, E. \& Metzler, R. Noisy continuous time random walks. J. Chem Phys. 139, 121916 (2013)

52. Ball, P. More than a bystander. Nature 478, 467-468 (2011).

53. Grossman, M. et al. Correlated structural kinetics and retarded solvent dynamics at the metalloprotease active site. Nat. Struct. Mol. Biol. 18, 1102-1108 (2011).

54. Guigas, G. \& Weiss, M. Sampling the cell with anomalous diffusion-the discovery of slowness. Biophys. J. 94, 90-94 (2008).

55. Klauda, J. B. et al. Update of the charmm all-atom additive force field for lipids: validation on six lipid types. J. Phys. Chem. B 114, 7830-7843 (2010).

56. MacKerell, A. D. et al. All-atom empirical potential for molecular modeling and dynamics studies of proteins. J. Phys. Chem. B 102, 3586-3616 (1998).

57. Jorgensen, W. L., Chandrasekhar, J., Madura, J. D., Impey, R. W. \& Klein, M. L. Comparison of simple potential functions for simulating liquid water. J. Chem. Phys. 79, 926 (1983).

58. Kalé, L. et al. Namd2: Greater scalability for parallel molecular dynamics. J. Comput. Phys. 151, 283-312 (1999).

\section{Acknowledgments}

This work is supported by the Core Research for the Evolution Science and Technology (CREST) of the Japan Science, Technology Corporation (JST), Keio University Program for the Advancement of Next Generation Research Projects, and MEXT Grant-in-Aid for the "Program for Leading Graduate School". 


\section{Author contributions}

E. Y., T. A., and K. Y. performed the calculation. The research reported here emerged from lively discussions between E. Y. and T. A., M. Y., and K. Y. All authors contributed to write the manuscript

\section{Additional information}

Supplementary information accompanies this paper at http://www.nature.com/ scientificreports

Competing financial interests: The authors declare no competing financial interests.
How to cite this article: Yamamoto, E., Akimoto, T., Yasui, M. \& Yasuoka, K. Origin of subdiffusion of water molecules on cell membrane surfaces. Sci. Rep. 4, 4720; DOI:10.1038/ srep04720 (2014)

(c) (i) $\Theta$ This work is licensed under a Creative Commons Attribution-NonCommercialNoDerivs 3.0 Unported License. The images in this article are included in the article's Creative Commons license, unless indicated otherwise in the image credit; if the image is not included under the Creative Commons license, users will need to obtain permission from the license holder in order to reproduce the image. To view a copy of this license, visit http://creativecommons.org/licenses/by-nc-nd/3.0/ 